\title{
A search for pulsations in two Algol-type systems V1241 Tau and GQ Dra
}

\author{
Burak Ulaş ${ }^{1}$, Ceren Ulusoy ${ }^{2}$, Kosmas Gazeas ${ }^{3}$, Naci Erkan ${ }^{4}$, \\ and Alexios Liakos ${ }^{5}$ \\ ${ }^{1}$ İzmir Turk College Planetarium, 8019/21 sok., No: 22, İzmir, Turkey \\ email: bulash@gmail.com \\ ${ }^{2}$ College of Graduate Studies, University of South Africa, PO Box 392, UNISA 0003, Pretoria, \\ South Africa \\ email: cerenuastro@gmail.com \\ ${ }^{3}$ Department of Astrophysics, Astronomy and Mechanics, National and Kapodistrian \\ University of Athens, GR-157 84, Zografos, Athens, Greece \\ email: kgaze@phys.uoa.gr \\ ${ }^{4}$ Department of Physics, Faculty of Arts and Sciences, Çanakkale Onsekiz Mart University, \\ Terzioglu Campus, TR-17100, Çanakkale, Turkey \\ email: nacierkan@comu.edu.tr \\ ${ }^{5}$ Institute for Astronomy \& Astrophysics, Space Applications \& Remote Sensing, National \\ Observatory of Athens, I.Metaxa \& Vas. Pavlou St., GR-15236, Palaia Penteli, Greece \\ email: alliakos@phys.uoa.gr
}

\begin{abstract}
We present new photometric observations of two eclipsing binary systems, V1241 Tau and GQ Dra. We use the following methodology: initially, the Wilson-Devinney code is applied to the light curves in order to determine the photometric elements of the systems. Then, the residuals are analysed using Fourier techniques. The results are the following. One frequency can be possibly attributed to a real light variation of V1241 Tau, while there is no evidence of pulsations in the light curve of GQ Dra.
\end{abstract}

Keywords. stars: binaries: eclipsing, stars: oscillations (including pulsations)

\section{Introduction}

V1241 Tau (= WX Eri) was found to be an eclipsing binary by Henrietta Leavitt (Pickering 1908). Sarma \& Abhyankar (1979) claimed that the primary component shows periodic variations in its light. However, the periods of this variation were simply $1 / 5$ and $1 / 6$ of the orbital period which is likely a result of the rectification process they applied. Arentoft et al. (2004) found no trace of pulsations. The light variation of GQ Dra was discovered by Hipparcos (ESA 1997).

\section{Observations and solution of the light curves}

$B V R I$ light curves of V1241 Tau were obtained with the 0.4-m telescope of the University of Athens Observatory in November 2012. GQ Dra was observed with the 1.22-m telescope of the Onsekiz Mart University Observatory on 7 nights in March and April 2013. Light curves of both systems were analysed using PHOEBE (Prša \& Zwitter 2005) software. The results are the following. V1241 Tau has a semi-detached configuration with the inclination of about $81.5^{\circ}$ and the mass ratio of 0.44 . The hotter and cooler components have the effective temperatures equal to $7500 \mathrm{~K}$ and $4906 \mathrm{~K}$, respectively. We find that 91 percent of the light in $V$ filter comes from the primary component. 

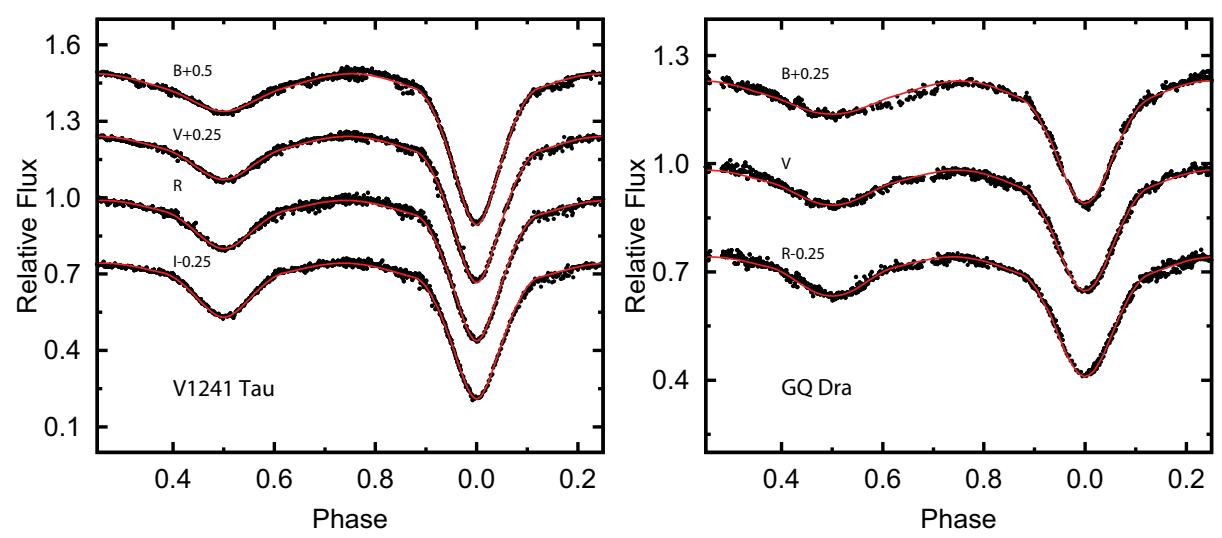

Figure 1. Observed (points) and theoretical (lines) light curves of the two systems. Some curves are shifted in flux axis for the sake of clarity.

Our solution for the semi-detached binary GQ Dra is the first light curve solution in the literature. Mass ratio of the system is found to be 0.25 and the orbital inclination was calculated to be $75.3^{\circ}$. The effective temperature of the secondary was found to be equal to $5050 \mathrm{~K}$ while the primary's $T_{\text {eff }}$ was fixed at $8750 \mathrm{~K}$. The comparison between observations and model light curve is shown in Fig. 1.

\section{Search for pulsations}

We searched the residuals from the PHEOBE fits for periodic variability using Fourier techniques. In the case of V1241 Tau, we found a significant peak at frequency of $f=$ $2.13 \mathrm{~d}^{-1}$ and amplitude of 0.0097 mag which possibly can be attributed to real changes in one of the components. However, it is worth to emphasize that the variability can be also due to instrumental effects. The same method was applied to the residuals from the solution of the light curve of GQ Dra. No remarkable periodic variability was found.

\section{Conclusions}

The main result regarding the pulsational variability in the systems is that there is no convincing evidence of pulsations in either of them. The residual light curve of the system V1241 Tau shows periodic variation with a small amplitude, but its origin is probably an instrumental effect.

\section{Acknowledgements}

CU sincerely thanks the South African National Research Foundation (NRF) for the award of NRF Multi-Wavelength Astronomy Research Programme (MWGR), Grant No: 86563 to Prof. L.L. Leeuw at UNISA, Reference: MWA1203150687.

\section{References}

Arentoft, T., Lampens, P., Van Cauteren, P., Duerbeck, H. W., García-Melendo, E., \& Sterken, C. $2004, A \mathscr{E} A, 418,249$

ESA, 1997, The Hipparcos \& Tycho Catalogues, ESA SP-1200

Pickering, E. C. $1908, A N, 178,157$

Prša, A. \& Zwitter, T. 2005, ApJ, 628, 426

Sarma, M. B. K. \& Abhyankar, K. D. 1979, Ap\&SS 65, 443 